\title{
Pediatric intracranial aneurysms
}

\author{
L. N. Tripathy, S. N. Singh \\ Department of Neurosurgery, Apollo Gleneagles Hospitals, Kolkata - 700 058, West Bengal, India
}

\author{
Address for correspondence: \\ Dr. L. N. Tripathy, \\ Department of Neurosurgery, \\ Apollo Gleneagles Hospitals, \\ Kolkata - 700 058, West Bengal, \\ India \\ E-mail: laxmitripathy@yahoo.co.uk
}

PMID: 19934568

DOI: $10.4103 / 0028-3886.57815$

\begin{abstract}
The incidence of subarachnoid haemorrhage from intracranial aneurysms in the paediatric age group is extremely rare. Interestingly, occurrence of vasospasm has been reported to be less in comparison to the adults. Both coiling and clipping have been advocated in selected cases. Because of the thinness of the wall of the arteries, utmost care should be taken while handling these arteries during surgery. The overall results of surgery in children have been reported to be better than their adult counterparts. We present four such cases from our own experience. All these children were operated upon, where the solitary aneurysm in each case was clipped and all of them made a good recovery.
\end{abstract}

Key words: Cerebral aneurysms, paediatric age group, clipping

\section{Introduction}

Aneurysmal subarchnoid hemorrhage (SAH) is uncommon in pediatric age group. Intracranial aneurysms account for 2-6\% of all intracranial aneurysms in this age group and the reported frequency of multiple aneurysms is also very low. ${ }^{[1]}$ More commonly the aneurysms are in the posterior circulation. ${ }^{[2]}$ Though clipping is the gold standard treatment option, endovascular treatment (coiling) is also evolving as an alternative option in this age group. ${ }^{[4]}$

\section{Case Reports}

Of the 268 intracranial aneurysms in the author's experience, only four were in the pediatric age group [Table 1].

\section{Case 1}

A 4-year-old girl presented with sudden onset severe headache, vomiting and right-sided limb weakness and speech difficulties. Computed tomography (CT) scan of brain showed SAH in the left sylvian fissure.
Magnetic resonance angiography (MRA) [Figure 1] and a four vessel cerebral digital subtraction angiogram (DSA) showed left middle cerebral artery (MCA) bifurcation aneurysm. She had clipping of the aneurysm a left pterional craniotomy and the postoperative period was uneventful. The neurological deficits recovered completely over a period of six months and her neurological status was normal at one year of post-operative follow-up.

\section{Case 2}

A 11-year-old male child was admitted with sudden onset headache and loss of consciousness. He rapidly deteriorated after admission. An emergency CT scan showed left cerebellar hematoma with acute hydrocephalus. An emergency external ventricular drain (EVD) was planned. On the operation table, he had ventricular tachycardia and was revived with DC shock and cardiopulmonary resuscitation. Following the placement of EVD, his cardiac rhythm remained normal. In the same sitting the cerebellar hematoma, was evacuated by a suboccipital craniectomy. At surgery, on opening the dura, the cerebellum was tense. There were

\section{Table 1: Clinical characteristics, surgical management, and outcome}

\begin{tabular}{lllll}
\hline Age of patient (in years) & Sex & Site of aneurysm & Treatment & Result \\
\hline 4 & Female & Left MCA [Figure 1] & Clipping & Good (at six months follow-up) \\
11 & Male & Left PICA* [Figure 2] & Clipping + EVD & Good (at three months follow-up) \\
11 & Male & Left MCA distal [Figure 3] & Clipping & Good with residual deficit (at two years follow-up) \\
14 & Male & ACom A [Figure 4] & Clipping & Good (at six weeks follow-up)
\end{tabular}

MCA - Middle cerebral artery; PICA - Posterior inferior cerebellar artery; EVD - External ventricular drainage; ACom A - Anterior communicating artery;

* - Associated arterio-venous malformation 
multiple blood vessels with arterialized veins suggestive of arteriovenous malformation (AVM) and there was also a giant right distal posterior inferior cerebellar artery (PICA) aneurysm. The clot was evacuated and the aneurysm was clipped with two straight long (17 mm) clips. The AVM was left behind untouched. He did a remarkable recovery after surgery. Postoperative cerebral angiography [Figure 2] confirmed a large AVM in the posterior fossa fed mainly by right vertebral artery. Patient was discharged after two weeks of surgery. At six weeks of follow-up, he was independent and had minimal cerebellar signs. Patient was advised endovascular treatment for the AVM, but unfortunately he was lost to follow-up.

\section{Case 3}

A 11-year-old boy presented to the Accident and Emergency Department with history of repeated vomiting, headache and altered mental status from the night prior to admission. Clinical examination revealed dilated left pupil with sluggish reaction

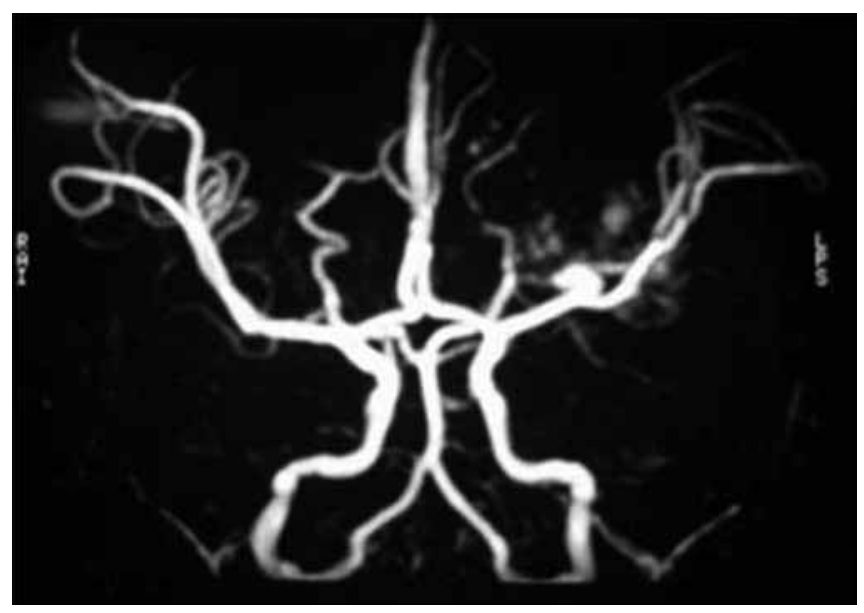

Figure 1: Post-operative cerebral digital subtraction angiogram (lateral view) showing obliteration of the distal left middle cerebral artery aneurysm

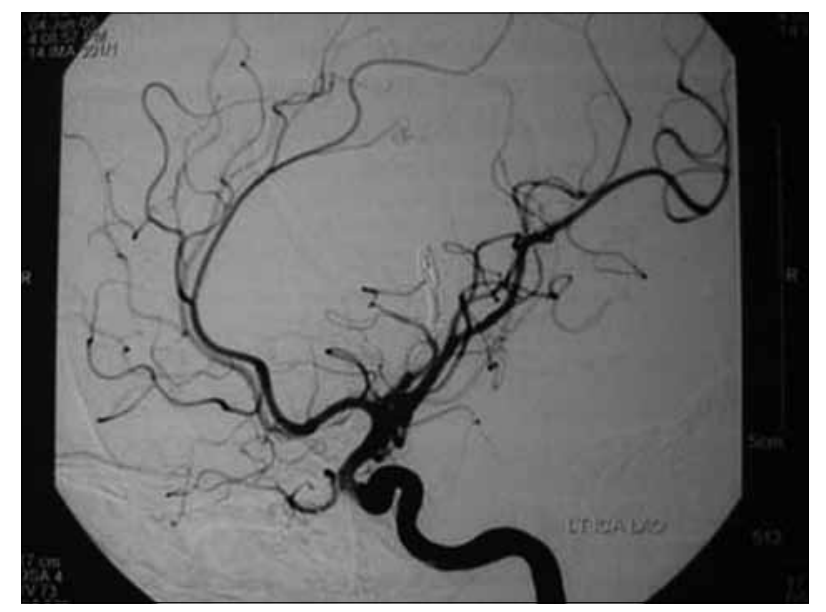

Figure 3: Cerebral angiogram (lateral view) showing the anterior communicating artery aneurysm to light and right sided weakness with extensor posturing to painful stimulus CT brain revealed left perisylvian intracerebral hematoma with midline shift. He was taken up for hematoma evacuation by a left temporo-parietal craniotomy. At surgery, after removal of the hematoma, a small aneurysm was seen on the left distal middle cerebral artery. The aneurysm was clipped. In the postoperative period, patient regained consciousness, but remained aphasic with right hemiparesis, which subsequently improved slowly. A postoperative cerebral angiogram [Figure 3] revealed normal cerebral vasculature with obliteration of the aneurysm. He is being followed up at regular interval in the outpatient clinic. At the last follow-up he has still residual weakness. He has gone back to school and learnt to write with left hand, prior to the ictus he was right handed.

\section{Case 4}

A 14-year-old boy presented with severe headache, following which he was remaining quiet and withdrawn

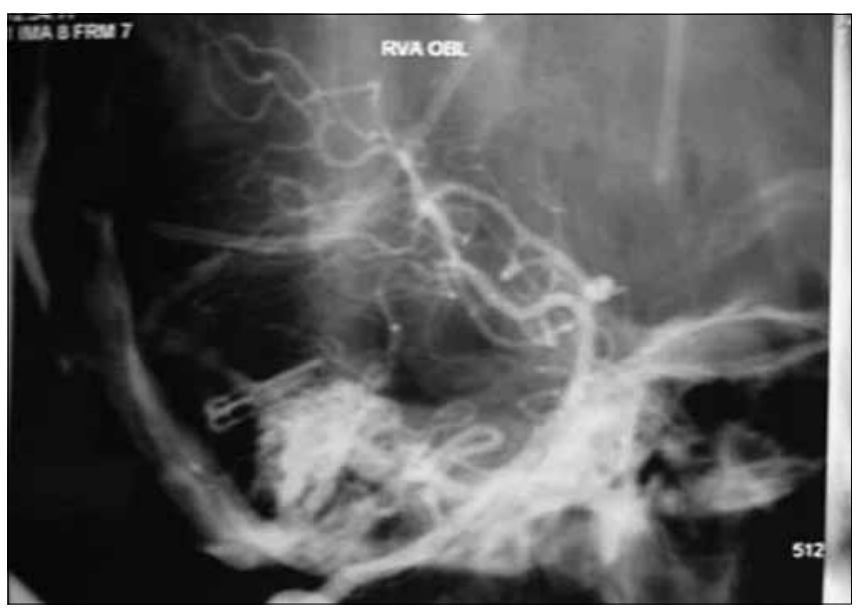

Figure 2: Post-operative cerebral angiogram (lateral view) showing the atriovenous malformation and two aneurysm clips with obliteration of the aneurysm arising from the right posterior inferior cerebellar artery

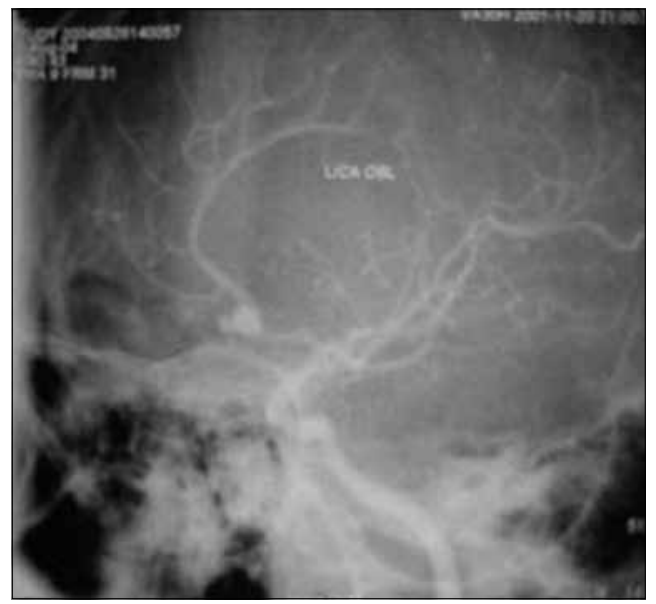

Figure 4: Magnetic resonance angiogram showing left middle cerebral artery bifurcation aneurysm 
from his surroundings. Initially he was thought to be suffering from a psychological disorder, but in a CT scan, there was evidence of SAH with the blood, present mostly in the anterior interhemispheric fissure. The cerebral angiography [Figure 4] revealed an aneurysm in anterior communicating artery (ACom A). Through a right frontotemporal craniotomy, the aneurysm was clipped. The postoperative period was uneventful. He had a complete recovery without any residual deficit. His initial follow-up at six weeks from surgery was unremarkable and his higher mental functions were improving.

Intracranial aneurysms in the pediatric age group pose considerable diagnostic and therapeutic challenges. The delay in the diagnosis is mainly due to the rarity of intracranial aneurysm in this age group and lack of awareness among the practicing paediatricians. Large size of the aneurysm and location in the posterior circulation pose difficulties for the treating clinician. ${ }^{[3]}$ The reported incidence of vasospasm in aneurismal $\mathrm{SAH}$ is low in paediatric age group is low. ${ }^{[3]}$ The possible explanations include elasticity of the cerebral vasculature in this age group and well preserved cerebral autoregulation. Although surgical techniques are standard and similar to those in adults, handling of smaller and delicate vessels require utmost care and precision. Of late, endovascular treatment has also been advocated for aneurysms in this age group with good success. ${ }^{[4]}$ Long-term follow-up data are necessary to understand the natural history of this disease. ${ }^{[5]}$

It is quite challenging for the neurosurgeon to operate on tiny arteries of a delicate and growing brain. Similar are the challenges for the interventional neuroradiologists to navigate through thin arteries and very thin-walled aneurysms for both diagnostic angiography and endovascular treatment.

\section{References}

1. Aryan HE, Giannotta SL, Fukushima T, Park MS, Ozgur BM, Levy ML. Aneurysms in children: Review of 15 years experience. J Clin Neurosci 2006;13:188-92.

2. Amacher LA, Drake CG. Cerebral artery aneurysms in infancy, childhood and adolescence, (16 cases). Childs Brain 1975;1:72-80.

3. Sharma BS, Sinha S, Mehta VS, Suri A, Gupta A, Mahapatra AK. Pediatric intracranial aneurysms-clinical characteristics and outcome of surgical treatment. Childs Nerv Syst 2007;23:327-33.

4. Lustgarten L, John T, Lopez R, Walzer I I, Kerr RS, Molyneux AJ, et al. Paediatric intracranial aneurysms: Results of a surgical series and literature review of Guglielmi detachable coil embolization. J Clin Neurosci 1999;6:133-7.

5. Jain PK, Mehta VS. Anterior communicating artery aneurysm in a 3-year-old girl. Childs Nerv Syst 2002;18:71-3.

Accepted on 02-07-2009

Source of Support: Nil, Conflict of Interest: None declared. 